\title{
Bandwidth Adaptive Hierarchies for On-Line ATM Quality of Service Routing
}

\author{
Steven Willmott ${ }^{1}$ and Boi Faltings \\ Laboratoire d'Intelligence Artificielle, \\ Ecole Polytechnique Federale de Lausanne, \\ Switzerland.
}

\begin{abstract}
On-line QoS routing in ATM networks involves challenging problems in the distribution of information and control. This paper presents a hierarchical routing architecture which adapts its own structure over time to match available bandwidth in the network. The adaptive hierarchy is shown to significantly outperform a similar static hierarchy in preliminary tests and comparisons with centralised and hop-by-hop routing approaches are also encouraging. The paper also discusses the relevance of matching the information model to the network state for standard (PNNI) hierarchical routing architectures.
\end{abstract}

\section{Introduction}

This paper focuses on routing Quality of Service (QoS) constrained connection demands in ATM networks. In particular we address the problem of designing routing architectures which support on-line, state based routing and make no statistical assumptions about traffic arrival patterns. This is known to be a complex problem $[10,11]$ and poses significant difficulties in both information distribution (representing/transmitting network state information) and control distribution (how to make path choices). As networks increase in size, the amount of state data that needs to be maintained and communicated throughout the network increases rapidly. Hierarchical architectures are one possible approach to the on-line QoS routing problem which mitigates this effect [2], however they invariably run into complex problems related to state information aggregation.

This paper re-examines hierarchical QoS routing and describes a routing hierarchy which adapts its own structure to the changing network state over time (Section 2) and compares this with generic static hierarchies in preliminary tests (Section 3). The paper also covers relevance to current practice in QoS routing with discussion of the relationship to standard hierarchical routing approaches (Section 4.3) and test comparisons with a centralised route server and hopby-hop approaches (Section 3.1.5 and 3.1.4 respectively). The adaptive hierarchy is shown to significantly outperform a generic hierarchical structure in distributed simulations.

\section{Routing Hierarchies}

Hierarchies have long been suggested as a way to improve both the scalability and robustness of routing [2] and other management tasks [1,7]. With the division between EGP routing and IGP's, the Internet effectively has a three level architecture, OSPF has two levels and the ATM Forum's PNNI architecture [5] also takes the form of a hierarchy.

The standard notion of a hierarchy in hierarchical routing refers to the information model only. Most approaches use a non-hierarchical control model (such as source or distributed routing for example) for route selection. However there are also potential benefits in linking the control model to the information model to make both hierarchical (defined as an i/c-hierarchy in Section 2.1 - "i/c" for "information/control" indicating that both the information and control models are strictly hierarchical). It is not our purpose to advocate $\mathrm{i} / \mathrm{c}$-hierarchical routing in this paper (this question involves multiple complex tradeoffs) but since the adaptive hierarchy uses an i/c model, Section 2.1 first defines a static i/c-hierarchy. Furthermore results from the static $\mathrm{i} / \mathrm{c}$ routing hierarchy are also very relevant to standard hierarchical routing models where only the information model is hierarchical (i-hierarchies - see Section 4.3).

\footnotetext{
${ }^{1}$ Author for correspondence: willmottelia.di.epfl.ch
} 


\subsection{Static i/c-Hierarchy (SH)}

Given a network (with sets of nodes $\mathrm{N}$ and links L, such that the state of each link $\mathrm{L}$ is described by a tuple valued quality vector $\mathrm{LiQV}$ ) a strict $i /$ c-hierarchy is defined as follows:

1. The network is divided up into disjoint regions $\mathfrak{R}_{l}$ with each region $\mathrm{r} \in \mathfrak{R}_{l}$ grouping together one or more network nodes. Each ii is managed by a controller ci which has precise knowledge of the network state in $r_{i}$,

2. The regions in $\Re_{1}$ are grouped together at the next level up into meta-regions $\left(r \in \Re_{2}\right)$ each controlled by a parent controller. Each of the controllers in $\Re_{1}$ presents a summarised representation of the network state in their region to their respective parent controllers. Each controller of a region in $\Re_{2}$ thus has an aggregate view of a larger part of the network composed of logical nodes representing a subset of the regions in $\Re_{1}$,

3. This hierarchical clustering is continued recursively to form a hierarchy covering the whole network. This generates a finite number of levels and regions if each region in $\Re_{i+1}$ clusters at least two regions in $\Re_{\text {. }}$.

Unicast demands di (each described by a quality vector $\mathrm{diQV}_{\mathrm{Q}}$ ) arise randomly according to an unknown distribution $\Omega$ and must be allocated a path through the network which satisfies $d_{i} Q V$.

4. When a demand $d_{i}$ is generated at a source node in a unique $r_{i} \in \Re_{1}$, the controller of $r_{i}$ tries to route the demand. If $\mathrm{d}_{\mathrm{i}}$ 's endpoint is known (i.e. also local to ri) and sufficient resources for $\mathrm{diQV}$ can be allocated, a route is assigned and the resources reserved (if sufficient resources are not available the demand is rejected). If di's endpoint is not local to $\mathrm{r}_{\mathrm{i}}$ then $\mathrm{c}_{\mathrm{i}}$ passes the demand up to its parent at level $\Re_{2}$ who is then responsible for coordinating route finding between the controllers in the regions in $\mathfrak{R}_{1}$ regions under its control,

5. This "passing of the buck" up the hierarchy continues recursively. The process terminates since at some level $\mathfrak{R}_{\mathrm{j}}$ both the start and endpoints of $d_{i}$ will fall into the same region and the number of levels is finite.

This definition of a hierarchy deliberately separates the information model (points 1-3) and the control model (points 4 and 5). Both information and control are strictly hierarchical and there is no direct communication (information or routing task exchange) between peers in the hierarchy. Routing is carried out by region controllers. In our experiments, controllers use simple delay constrained shortest path algorithms having first filtered out links with insufficient bandwidth capacity (i.e. delay and bandwidth are the only QoS parameters treated).

\subsection{Adaptive i/c-Hierarchy (AH)}

Even with this strict definition of a hierarchy, there is no unique form for a given network topology and it is not clear how to choose one which is likely to be efficient. Furthermore, in an environment as dynamic as a communications network, no single hierarchical decomposition is optimal for all network states. One solution is therefore to allow the hierarchical structure itself to adapt over time - allowing controllers in the hierarchy to update their roles, scope of knowledge, and relationships to others.

The key points of the adaptive routing architecture are as follows:

- $\quad$ Starting from the definition in Section 2.1, the regions in the adaptive hierarchy are defined in terms of the residual bandwidth available for incoming communication demands on the links in the network. More precisely, given a communication bandwidth requirement ( $\beta$ Mbit/s), network nodes can be clustered into equivalence classes of nodes which are mutually reachable at bandwidth $\beta$ Mbit/s (this assumes symmetric resource availability on links). Equivalence classes are formed because reachability over a convex metric is a transitive relation. Each equivalence class is then considered a region $r \in \Re_{\beta}$,

- This clustering repeats at all levels of the hierarchy with $\beta$ decreasing at successively higher levels. The lowest level $\Re_{\beta 1}$ ) therefore contains the smallest regions, clustering nodes connected by very high available bandwidth. Regions at higher levels are connected at progressively lower available bandwidths.

A more complete description can be found in [12]. The approach gives a unique network decomposition (and hence hierarchy $A H(R)$ ) for any network state $R$ (see [3] for a proof). As the bandwidth resources on the links change over time (due to allocations and deallocations of network traffic) controllers detect this and update their organisational relationships by merging and splitting their regions: 
- Merge: As resources in one area of the network (on a single link) become available, controllers merge their regions to reflect new stronger connectivity,

- Split: As resources are allocated to traffic demands, controllers divide their efforts and split regions into two or more new regions (each with their own controller).

Together, these two operations allow the organisation to tend to the well defined, unique, state $A H(R)$. Hence, using only local decision criteria, the region controllers adapt the hierarchy to the network state $R$ they are routing demands in.

\section{$3 \quad \begin{aligned} & \text { Testing and Preliminary } \\ & \text { Results }\end{aligned}$}

Experiments were carried out on a fully distributed Java test bed made up of one Java Virtual Machine per simulated network node with nodes communicating via TCP sockets (appropriately delayed to simulate network communication delay). Node controllers implement full ATM PNNI connection setup protocol suite.

The tests described in this paper were carried on two network topologies:

- T1: a model of the Compuserve USA backbone which has 11 nodes and 14 (45Mbit/s) ATM links (Source: http://www.caida.org/Tools/Mapnet/),

- T2: a (slightly modified) model of the Cable and Wireless USA backbone which has 18 nodes and 30 ATM links ranging from $622 \mathrm{Mbit} / \mathrm{s}$ to $2.4 \mathrm{Gbit} / \mathrm{s}$ http://www.caida.org/Tools/Mapnet/.)

(Source:

Traffic arrival patterns for the test runs were generated such that each node had an equal probability of generating demands to any other node. The delay requirement was fixed at 1.25 times the maximum delay across the network via the shortest path (this effectively means that delay is only factor when many shortest routes are not available). The demand arrival rates were varied over the test period, increasing over time.

In each case tests were carried out for:

- $\quad$ SH: The static i/c-hierarchy defined in Section 2.1 ,

- $\mathbf{A H}$ : The adaptive i/c-hierarchy defined in Section 2.2,
- CRS: A centralised route server, running a simple shortest path algorithm which takes into account bandwidth availability and delay only,

- HBH: A simple hop-by-hop scheme which routes demands along the a-priori shortest path by delay and is not adaptive to the network state.

Experiments were carried out in accelerated time simulating 10 hours of network operation in 1 hour of real time for T1, 10hours of network operation in 2 hours for T2 (all values in the results sections below are given w.r.t. simulated time).

\subsubsection{Results for $\mathrm{T} 1$}

The traffic parameters used for testing on the T1 Compuserve model were:

- Demand hold times were evenly distributed between 400 and 800 seconds,

- Bandwidth requirements evenly distributed between 1 and $10 \mathrm{Mbit} / \mathrm{s}$ per demand,

- Demand arrival rate starting at an average of 12 demands per hour per node and increasing steadily to an average rate of 36 demands per hour per node.

- Experiments ran for 10 hours simulated time.

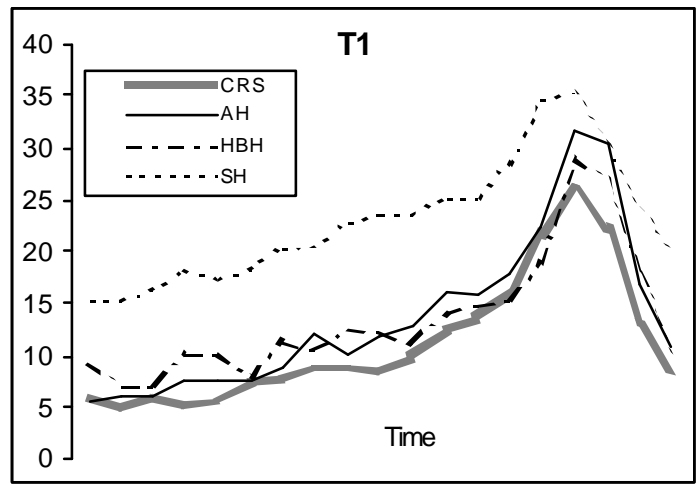

Fig. 1 T1 (rejection \%age v's time): AH rejects significantly less demands that $\mathrm{SH}(13 \%$ average v's $22 \%$ average over the whole period). CRS and $\mathrm{HBH}$ drop an average of $11 \%$ of their demands over the period. Standard deviations (for variance between experiment runs) vary between 3\% and 9\% (larger variances at increased traffic load).

Figure 1 summarises rejection percentages for all four approaches for the 10 hour experiment period, each averaged over 16 test runs. Comparisons using the percentage of offered bandwidth accepted give comparable results. The results show that even 
though both hierarchies ( $\mathrm{SH}$ and $\mathrm{AH}$ ) had access to the same state information and worked under the same network conditions, AH performs significantly better than SH.

\subsubsection{Results for $\mathrm{T} 2$}

The traffic parameters used for testing on the T2 model were:

- Demand hold times were evenly distributed between 600 and 1800 seconds,

- Bandwidth requirements evenly distributed between 10 and $110 \mathrm{Mbit} / \mathrm{s}$ per

- Demand arrival rate starting at an average of 2 demands per hour per node and increasing steadily to an average rate of 12 demands per hour per node.

- Experiments ran for 10 hours simulated time.

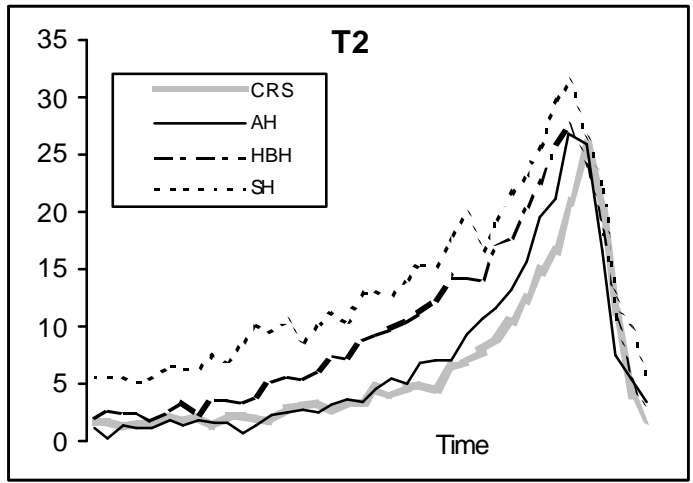

Fig. 2 T2 (rejection \%age v's time): Over ten runs SH rejects $13 \%$ on average, $\mathrm{HBH} 10 \%, \mathrm{AH} 7 \%$ and CRS 6\%.

Figure 2 summarises rejection percentages for all four approaches averaged over 10 test runs. Comparisons using the percentage of offered bandwidth accepted give comparable results.

\subsubsection{Comparing SH and AH}

AH performs significantly better in both test sets and across the whole period of operation as the network load increases. The performance of the AH does drop more sharply near the traffic peak (point of highest load / demand arrival rate) however. This seem to be due to two factors:

1. The network becoming saturated and both schemes are dropping large numbers of demands,
2. The increased dynamicity of the network increasing AH's cost of adaptation (see Section 4.2).

It is significant however that, despite this, AH still outperforms $\mathrm{SH}$ by a considerable margin. The advantage of $\mathrm{AH}$ over $\mathrm{SH}$ appears to be even greater in the larger network with AH's performance almost equalling that of CRS. This result is encouraging since it indicates potential scalability in $\mathrm{AH}$ type approaches.

\subsubsection{Comparison with HBH}

Results for $\mathrm{HBH}$ are comparable to $\mathrm{AH}$ and CRS in the 11 node $\mathrm{T} 1$ network but are considerably worse in the larger T2 network. This appears to be mainly due to the increased size of the network core in T2. Since $\mathrm{HBH}$ does not use the network state to make routing decisions, it sends proportionally more traffic via direct routes through the centre of the network which leads to heavy congestion whilst routes avoiding the network centre are left unused.

$\mathrm{AH}$ and CRS are both able to make better use of network resources by relying on network state information to avoid congested areas. Although this primarily confirms the utility of state based routing in QoS networks it also shows $\mathrm{AH}$ beginning to outperform standard benchmark approaches.

\subsubsection{Comparison with CRS}

AH's performance is only slightly worse than CRS' in both tests, with results very close in the T2 tests. Although comparison of raw percentages is somewhat misleading given the variances between individual test runs, this does indicate that $\mathrm{AH}$ and CRS performances are very similar. Performance is particularly close when the network is lightly loaded.

The similarity in performance in the lightly loaded case is partly due to the similarity of AH's structure to CRS in this network resource configuration. Since the number and distribution of disjoint regions in $\mathrm{AH}$ depends upon the bandwidth availability in the network, $\mathrm{AH}$ has relatively few regions in a lightly loaded network (3/4 regions at the two lower levels on average in $\mathrm{T} 1$ and $\mathrm{T} 2$ ). This configuration is therefore similar to CRS which effectively acts a single large region.

A final important thing to note in the comparison is that CRS has "complete information" about the network state. This is manageable in a small network but unrealistic in larger networks and it is encouraging 
that AH's performance is comparable since hierarchical schemes such as $\mathrm{SH}, \mathrm{AH}$ and PNNI are potentially more scalable in larger networks. Clearly however, as a potential alternative to current techniques $\mathrm{AH}$ still requires considerable development and (above all) testing in more varied traffic and network scenarios.

\section{Discussion}

At first glance it seems implausible that the adaptive hierarchy should outperform the static hierarchy by such a large margin. Both schemes have access to the same state information and use comparable information and control models. Section 4.1 outlines the main reasons behind this result, Section 4.2 covers the tradeoffs between adaptation and routing tasks and Section 4.3 discusses relevance to standard static hierarchical routing approaches.

\subsection{Comparison of $\mathrm{SH}$ and $\mathrm{AH}$}

As a demand is treated in both $\mathrm{SH}$ and $\mathrm{AH}$, it is pushed up to a level in the hierarchy where one controller has both nodes in its region (or they are clustered by its children). This controller then mediates the search for a path. If no path can be found at this level then the demand is rejected.

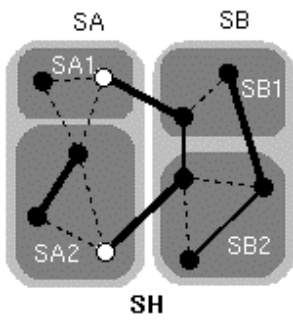

SH

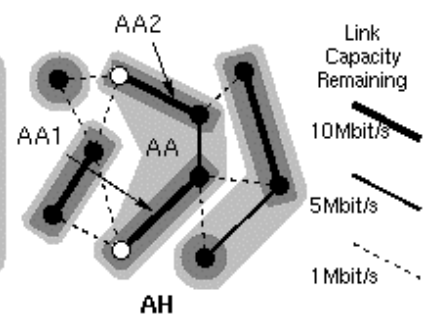

AH
Fig. 3 Both hierarchies cover the same network state and attempt to find a route between the two white nodes with $4 \mathrm{Mbit} / \mathrm{s}$ or more of bandwidth available end-to-end. The bandwidth values $1,5,10$ are taken as the values of $\beta$ (see Section 2.2) for the $\mathrm{AH}$ structure in the right hand diagram.

When searching for a route, the static hierarchy passes the task to the controller of region SA (left hand diagram in Figure 3) since each of the demand's endpoints are clustered by one of its immediate children. The controller of region SA $\left(\mathrm{c}_{\mathrm{SA}}\right)$ is however unable to find a route with sufficient bandwidth available because:
1. csa knows of no route between its relevant child regions (rsa1 and 15A2) with sufficient capacity available,

2. Without extra state information (about bandwidth availability internal to rsA1 and rsa2), it is also impossible for CSA to know whether the child regions have enough spare capacity available to locally route some section of any route it might propose.

Both of these facts show that that $\mathrm{SH}$ does not have convenient access to pertinent network state information - even though this information is represented within $\mathrm{SH}$ as whole. Adapted $\mathrm{AH}$ (right hand diagram in Figure 3) on the other hand, is able to find a route with the cooperation of just three controllers:

- AA's controller is guaranteed that at least one route for the demand exists within its own region since this region is connected at a bandwidth availability higher than that of the demand,

- Without the need for extra state data, $\mathrm{c}_{\mathrm{AA}}$ also knows that each of the region controllers in the level below ( $\mathrm{c}_{\mathrm{AA} 1}$ and $\mathrm{c}_{\mathrm{AA} 2}$ ) will be able to route their section of any route it suggests for the demand. This is because the clusters are defined connected at a higher bandwidth availability than rAA.

These two facts mean that once the demand arrives at a level in the hierarchy were it can be treated (i.e. both its endpoints are known) at least one local path with sufficient free bandwidth is guaranteed to be available. It should be noted that this guarantee only holds when the hierarchy is fully "up to date". In practice the hierarchy lags the uniquely defined distribution $A H(R)$ slightly as it adapts to the changing $R$. With its adaptation over time $\mathrm{AH}$ captures bandwidth state information in its own structure.

It would be possible to allow $\mathrm{SH}$ to search further by (for example) passing the demand to higher level in the hierarchy. This is difficult to achieve however since: it would require complex delegation model (involving peer to peer communication between regions) and, more importantly, it becomes very difficult to bound the search for a route (see Section 4.3). Ultimately, for $\mathrm{SH}$, solving the problem in the figure would involve all of the regions in the hierarchy. 


\subsection{The Impact of Organisational Dynamics}

As identified in [8], organizational change comes at a cost - the time given over to updating the organisation cannot be used for routing. To give some idea of the effect of the adaptation in the organisation, Figure 4 shows the adaptation activity over the period of one representative experiment run. As the demand arrival rate increases, the speed of change of $R$ (and hence of $A H(R))$ increases. This generates more stimulus for the controllers in the organisation to perform merges and splits to keep pace with $A H(R)$. The cost of adaptation was magnified by the fact that experiments were run at 10 times simulation time - resulting in potential peak traffic rates of up to 4000 demands per hour.

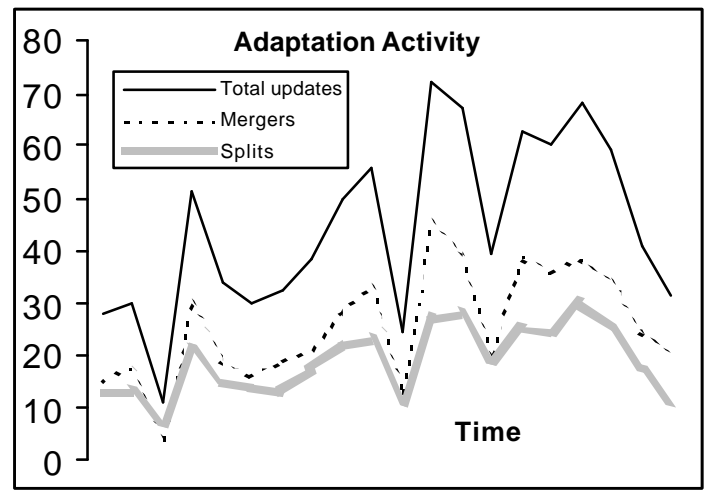

Fig. 4 For a typical experiment run, adaptation activity increases with the call arrival rate (and the load) in the network. (Note: splits total less than mergers since mergers are always binary whereas a region may split into 2 to $n$ new regions in one step.)

A second, more concrete, measure of the cost of adaptation is given by the number of demands rejected by controllers temporarily deciding to adapt the organisational structure rather than perform routing tasks. As is noted in Section 3.1.3, as the organisation undergoes more frequent change, losses due to adaptation do make themselves felt in the overall performance of the system. To manage the trade off between adaptation and problem solving, decisions to adapt are based on several factors:

- The agreement of peers. For a merger for example, one region controller must contact the controller of the region it would like to merge with to ensure the other controller is prepared to perform the action,

- The number of active routing tasks (which would have to be dropped if the organisational update goes ahead). This consideration balances the commitment to current routing tasks against the need to change to the structure to better facilitate future tasks,

- Damping factors (thresholds) which prevent immediate reaction to network state changes. A change in network state must therefore be significant or persist over a specified period before an agent will consider an update.

The number and level of the hierarchies (i.e. the choices of the $\beta$ values introduced in Section 2.2) also have a profound effect on the dynamism of the organisation. Choosing high values of $\beta$ (specifying strongly connected clusters) increases the dynamicity in the organisation and builds up more "structure" i.e. induces a greater number of smaller disjoint regions. Lower values of $\beta$ result in larger (and hence more stable) connected regions. The values used in the $\mathrm{T} 1$ experiments described here were 0,5 and 15 for example, giving a three tier hierarchy (the top level 0 is to ensure the whole never becomes disconnected). The values were chosen with experimentation and tests are underway to study the effects of varying these parameters. The number of levels was set at three and in general can be chosen by relating the value to the optimal decision tree for a hierarchy $\ln (\mathrm{n})$ levels with a branching factor of $\ln (n)$ where $n$ is the number of network nodes. For more on static clustering techniques see [6].

\subsection{Standard Hierarchical Routing (i- Hierarchies)}

As stated in Section 2, the term "hierarchical routing" is generally only applied to the information model ([9] and [5] for example). Hence the results obtained for $\mathrm{SH}$ are not directly comparable to those which might be expected of a PNNI architecture. (PNNI is selected for comparison here since it is the most relevant architecture for hierarchical QoS routing.)

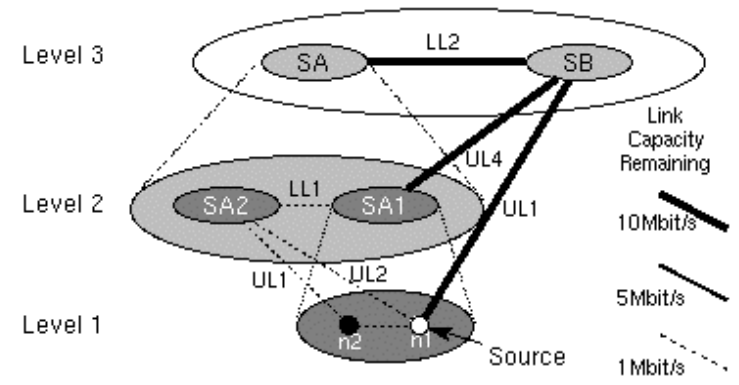

Fig. 5 Using the information model available to $\mathrm{SH}$ in Figure 3 makes the source node $n 1$ dependent on 
accurate models of SB, SA and SA1 to determine how best to reach the destination (which is in peer group SA2). LL denotes a logical link and UL denotes an uplink.

The results are still of relevance however, since PNNI relies on an information model generated by a static information hierarchy. Figures 5 and 6 show the PNNI information models for the $\mathrm{SH}$ and $\mathrm{AH}$ organisations generated by the network example in Section 4.1. Each shows the information model from the point of view of the source node (the task as before is to route a demand of $4 \mathrm{Mbit} / \mathrm{s}$ from the source $n 1$ to the destination - in SA2 and AA2 respectively). The diagram notation if borrowed from [5] with additions to show the residual bandwidth available on links (logical and physical).

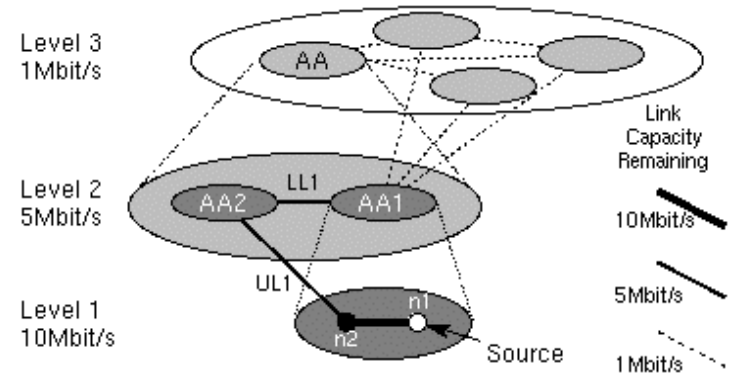

Fig. 6 Using the adapted $\mathrm{AH}$ information model, higher capacity links are close to the source and the nearby peer groups. (N.B. uplinks from level 1 to Level 3 - all at $1 \mathrm{Mbit} / \mathrm{s}$ - have been omitted for clarity.)

In SH's model, the source router must have accurate models for SB, SA, and SA2 to know that the path: $[n 1, \mathrm{SB}, \mathrm{SA}, \mathrm{SA} 2]$ is feasible. This highlights PNNI's dependency on complex aggregation techniques to represent logical nodes. Without a sufficiently detailed representation, the path could not be found. Techniques such as those discussed in [4] do make it possible to construct such models but detail must always be traded off against increasing the size of the representation. A full mesh complex node model, for example, may be larger than a faithful representation of the original network. SH's resulting path also passes through all peer groups in the hierarchy.

The effect of the sub-optimal information model is clearest when considering routing. Using the standard PNNI routing algorithm given in [5]:

1. The source node in the SH hierarchy (Figure 5) would generate the DTL $[n 1, \mathrm{SB}, \mathrm{SA}]$. Leaving border nodes in SB, SA and SA2 to complete the route,
2. The source node in the AH hierarchy (Figure 6) would generate the DTL $[n 1, n 2$, AA2]. Leaving a border node in AA2 to complete the route.

The SH DTL would be refused by the standard implementation of the algorithm in [5] which is designed to prevent loops ( $n l$ is in SA). This illustrates the conflict between bounding search and completeness. Even if the DTL is not refused, the source node must rely on SB's border node (information model shown in Figure 7) which receives the DTL to route the demand via SB2 rather than straight back to SA. Again, accurate models of SB2 and SA would be required to detect that going through SB2 would be preferable.

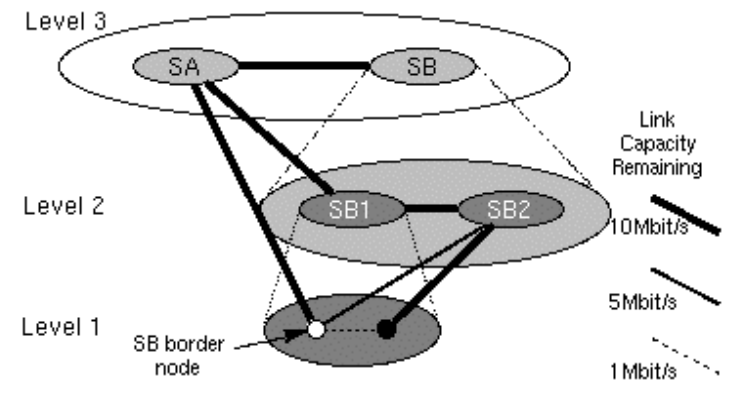

Fig. 7 The border node in SB's information model which first receives SH's DTL has little information on how to proceed.

In the routes generated using the $\mathrm{AH}$ information model, this kind of pathology cannot arise because the peer groups are a function of resources and not apriori boundaries. The same arguments as given in Section 4.1 apply:

- Uplinks within the parent peer group have by definition at least as much bandwidth available as those traversing the hierarchy (as link UL1 does in Figure 5),

- The source router in $\mathrm{AH}$ has the guarantee that AA2 is able to route all demands from any of its border nodes to any other of its nodes (including the destination) at up to $10 \mathrm{Mbit} / \mathrm{s}$ second.

Together these facts mean that if routes exist which traverse the hierarchy (in the way SH's route must), an internal route must also exist. The part of the information model relevant to the search is also strictly bounded to AA1, AA2 and AA since the clustering automatically indicates that nothing else is reachable above $1 \mathrm{Mbit} / \mathrm{s}$ (the demand requires $4 \mathrm{Mbit} / \mathrm{s}$ ). 
Comparing information models alone, PNNI's performance in small networks can be expected to fall between that of SH and CRS since:

- PNNI systems may employ extended logical node models $([4,5])$ and $\mathrm{SH}$ only uses a simple uniform node representation with no metrics applied,

- CRS has complete information about the network whilst PNNI must work with aggregate models.

From this, we would expect AH's performance to compare favourably with PNNI's in the types of network tested in scenarios $\mathrm{T} 1$ and $\mathrm{T} 2$ (Section 3) since AH is able to match CRS's performance closely despite being a hierarchical approach.

Unfortunately, direct comparison between $\mathrm{AH}$ and a PNNI architecture is difficult since 1) PNNI's implementation complexity is very high and 2) PNNI relies heavily on aggregation techniques and heuristics - for which many variants already exist. $\mathrm{AH}$ clearly requires more extensive testing:

- In larger networks,

- With heavier/more varied traffic loads (where the cost of organisational dynamics may have a greater impact).

It must also be noted that PNNI provides a large number of functionalities such as signalling, address summarisation and security (by hiding topology information inside peer groups) which the prototype $\mathrm{AH}$ does not. In fact, the last two functions (address summarisation and security) would both be complicated in $\mathrm{AH}$ by the need for the hierarchy to cluster arbitrary (w.r.t. administrative and naming domains) collections of nodes at any point in time.

\section{Conclusions}

With the current level of testing, work done so far can only be considered as a proof of concept and in-depth testing is now under way with larger networks, varied traffic matrices and other comparison metrics as well as the integration of standard techniques such as route caching and triggered state updates.

Despite the simplicity of the test scenarios however, results are encouraging:

- The adaptive hierarchy significantly outperforms the corresponding static hierarchy,
- The performance of the adaptive hierarchy is encouraging when compared with a centralised state based router with complete information,

- The poor correlation with the information model which SH suffers from also manifests itself in PNNI information models by increasing dependence upon state aggregation techniques for representing logical nodes.

In summary, routing performance is highly dependent on the availability of pertinent network state information. Allowing both the information and control structures to adapt to the network state provides a way of benefiting from the advantages of hierarchical routing whilst mitigating its dependence on information ag gre gation.

\section{$5.1 \quad$ Acknowledgements}

This work was carried out as part of the SPP-ICC IMMuNe project and its continuation funded by the Swiss National Science Foundation as project numbers SPP-ICC 5003-45311 and 21-59081.99 respectively.

\section{$6 \quad$ Literature}

[1] Bouloutas, A.; Gopal, P. M.: Clustering Schemes for Network Management. Networking in the 90s, volume 1. 1991, pp. 111-120.

[2] Chen, S.; Nahrstedt, K: An Overview of Quality of Service Routing for Next-Generation High-Speed Networks: Problems and Solutions. November/December 1998, IEEE Network, pp. 6479.

[3] Frei, C; Faltings, B.: Abstraction and Constraint Satisfaction Techniques for Planning Bandwidth Allocation. In proceedings IEEE INFOCOM'2000, Tel-Aviv, Israel, March 2000. IEEE proceedings in print.

[4] Lee, W. C.: Topology Aggregation for Hierarchical Routing in ATM Networks. In proceedings SIGCOMM, April 1995. pp 82-92.

[5] ATM-FORUM: P-NNI V1.0 - ATM Forum approved specification, af-pnni-0055.000. 1996.

[6] Ramamoorthy, C. V.; Srivastava, J.; Tsai, W-T.: Clustering Techniques for Large Distributed Systems. In proceedings IEEE INFOCOM'86. pages 395-404.

[7] Siegl, M. R.; Trausmauth, G.: Hierarchical Network Management: a Concept and its Prototype in SNMPv2. Journal of Computer Networks and ISDN Systems, 4/28. pp. 441-452. February 1996.

[8] So, Y-P.; Durfee, E. H.: Designing Organisations for Computational Agents. In "Simulating 
Organisations", Eds: Prietula, M. J.; Carley, K.

M.; Gasser, L. pp. 47-64. AAAI Press 1998.

[9] Tsai, W. T.; Ramamoorthy, C. V.; Tsai, W. K.; Nishiguchi, O.: An Adaptive Hierarchical Ro uting Protocol. IEEE Transactions on Computers 8/38. August 1989, pp. 1059-1075.

[10] Vedantham, S.; Iyengar, S. S.: The Bandwidth Allocation Problem in the ATM network model is NP-Complete. Information Processing Letters, volume 65, 1998. pp. 179-182.

[11] Wang, Z.; Crowcroft, J.: Quality-of-Service Routing for Supporting Multimedia Applications. IEEE Journal on Selected Areas in Communications 7/14. 1996.

[12] Willmott, S. N.; Frei, C.; Faltings, B.; Calisti, M.: Organisation and Co-ordination for On-line Routing in Communications Networks. In "Software Agents for Future Communication Systems". Eds: Hayzelden, A. L. G.; Bigham, J. pp. 130-159. Springer Verlag April 1999. 